\title{
New Approaches to Password Authenticated Key Exchange Based on RSA
}

\author{
Muxiang Zhang \\ Verizon Communications Inc., \\ 40 Sylvan Road, Waltham, MA 02451, USA \\ muxiang.zhang@verizon.com
}

\begin{abstract}
We investigate efficient protocols for password-authenticated key exchange based on the RSA public-key cryptosystem. To date, most of the published protocols for password-authenticated key exchange were based on Diffie-Hellman key exchange. It seems difficult to design efficient password-authenticated key exchange protocols using RSA and other public-key cryptographic techniques. In fact, many of the proposed protocols for password-authenticated key exchange based on RSA have been shown to be insecure; the only one that remains secure is the SNAPI protocol. Unfortunately, the SNAPI protocol has to use a prime public exponent $e$ larger than the RSA modulus $n$. In this paper, we present a new password-authenticated key exchange protocol, called PEKEP, which allows using both large and small prime numbers as RSA public exponent. Based on number-theoretic techniques, we show that the new protocol is secure against the e-residue attack, a special type of off-line dictionary attack against RSA-based password-authenticated key exchange protocols. We also provide a formal security analysis of PEKEP under the RSA assumption and the random oracle model. On the basis of PEKEP, we present a computationally-efficient key exchange protocol to mitigate the burden on communication entities.
\end{abstract}

\section{Introduction}

The design of authentication and key exchange protocol is usually based on the assumption that entities either share or own some secret data (called keys) which are drawn from a space so large that an adversary can not enumerate, either online or off-line, all possible keys in the space. In practice, however, cryptographic keys may often be substituted by human-memorable passwords consisting of only six to ten characters. The consequence is the proliferation of the so-called exhaustive guessing or dictionary attacks against many password-based systems [26]. Password guessing attacks have been around for so long, it seems paradoxical that strong authentication using only small passwords would be possible. In 1992, Bellovin and Merritt [5] showed that such paradoxical protocols did exist. They presented a family of protocols known as Encrypted Key Exchange, or EKE. By using a combination of symmetric and asymmetric (public-key) cryptographic techniques, EKE provides insufficient information for an adversary to verify a 
guessed password and thus defeats off-line dictionary attacks. Following EKE, a number of protocols for password-based authentication and key exchange have been proposed, e.g., [2, 6, 8, 10, 11, 13, 15-17, 21, 25] A comprehensive list of such protocols can be found in Jablon's research link [14].

Password-authenticated key exchange protocols are attractive for their simplicity and convenience and have received much interest in the research community. Over the last decade, many researchers have investigated the feasibility of implementing EKE using different public-key cryptosystems such as RSA, ElGamal, and Diffie-Hellman key exchange. Nonetheless, most of the well-known and secure variants of EKE are based on Diffie-Hellman key exchange. It seems that EKE works well with Diffie-Hellman key exchange, but presents subtleties when implemented with RSA and other public-key cryptosystems. In their original paper [5], Bellovin and Merritt pointed out that the RSA-based EKE variant is subject to a special type of dictionary attack, called e-residue attack. Based on number-theoretic techniques, Patel 22] further investigated the security of the RSA-based EKE variant and concluded that simple modifications of EKE would not prevent the RSA-based EKE variant from off-line dictionary attacks. In 1997, Lucks 18 proposed an RSA-based password-authenticated key exchange protocol (called OKE) which was claimed to be secure against the $e$-residue attack. Later, Mackenzie et al. 19] found that the OKE protocol is still subject to the $e$-residue attack. In [19], Mackenzie et al. proposed an RSA-based password-authenticated key exchange protocol (SNAPI) and provided a formal security proof in the random oracle model. The SNAPI protocol mandates that the public exponent $e$ be a prime number larger than the RSA modulus $n$. This ensures that $e$ is relatively prime to $\phi(n)$.

To avoid using large public exponents, Zhu et al. 27] proposed an "interactive" protocol which is revised from an idea of [5]. In the interactive protocol, Bob sends to Alice a number of messages encrypted using Alice's public key. If Alice can successfully decrypt the encrypted messages, then Bob is ensured that the encryption based on Alice's public key is a permutation. In [24, Wong et al. revised the interactive protocol of [27] to reduce the message size involved in the interactive protocol. Recently, Catalano et al. 9] proposed an interactive protocol similar to that of 24 and provided a security proof in the random oracle model. A drawback of the interactive protocols [27, 24, 9] is the large communication overhead involved in the verification of RSA public key.

In this paper, we investigate RSA-based password-authenticated key exchange protocols that can use both large and small primes as RSA public exponent, but without inducing large communication overhead on communication entities. For this purpose, we develop a new protocol for password-authenticated key exchange based on RSA. The new protocol, called PEKEP, involves two entities, Alice and Bob, who share a short password and Alice possesses a pair of RSA keys, $n, e$ and $d$, where $e d \equiv 1(\bmod \phi(n))$. Unlike the protocol SNAPI, however, the new protocol PEKEP allows Alice to select both large and small primes for the RSA public exponent $e$. In the protocol PEKEP, Bob does not need to verify if $e$ is relatively prime to $\phi(n)$, and furthermore, Bob does not 
have to test the primality of a large public exponent selected by Alice. Thus, the protocol PEKEP improves on SNAPI by reducing the cost of primality test of RSA public exponents. The protocol PEKEP also improves on the interactive protocols of [27, 24, 9] by reducing the size of messages communicated between Alice and Bob. Based on number-theoretic techniques, we prove that the protocol PEKEP is secure against the e-residue attack as described in [5, 22, We also provide a formal security analysis of PEKEP under the RSA assumption and the random oracle model.

To further reduce the computational load on entities, we present a computationally efficient key exchange protocol (called CEKEP) in this paper. The protocol CEKEP is derived from PEKEP by adding two additional flows between Alice and Bob. With the two additional flows, we show that the probability for an adversary to launch a successful $e$-residue attack against CEKEP is less than or equal to $\varepsilon$, where $\varepsilon$ is a small number (e.g., $0<\varepsilon \leq 2^{-80}$ ) selected by Bob. In the protocol CEKEP, the computational burden on Bob includes two modular exponentiations, each having an exponent of $\mathcal{O}\left(\left\lceil\log _{2} \varepsilon^{-1}\right\rceil\right)$ bits. When $\varepsilon=2^{-80}$, for example, the computational burden on Bob is lighter than that in a Diffie-Hellman based password-authenticated key exchange protocol. In the Diffie-Hellman based EKE variant, Bob needs to compute two modular exponentiations, each having an exponent of at least 160 bits.

The rest of the paper is organized as follows. We provide an overview of the security model for password-authenticated key exchange in Section 2. In Section 3, we present the protocol PEKEP and investigate its security against $e$-residue attack. We describe the protocol CEKEP in Section 4 and pursue formal security analysis of PEKEP and CEKEP in Section 5. We conclude in Section 6.

\section{$2 \quad$ Security Model}

We consider two-party protocols for authenticated key-exchange using humanmemorable passwords. In its simplest form, such a protocol involves two entities, say Alice and Bob (denoted by A and B), both possessing a secret password drawn from a small password space $\mathcal{D}$. Based on the password, Alice and Bob can authenticate each other and upon a successful authentication, establish a session key which is known to nobody but the two of them. There is present an active adversary, denoted by $\mathcal{A}$, who intends to defeat the goal for the protocol. The adversary has full control of the communications between Alice and Bob. She can deliver messages out of order and to unintended recipients, concoct messages of her own choosing, and create multiple instances of entities and communicate with these instances in parallel sessions. She can also enumerate, off-line, all the passwords in the password space $\mathcal{D}$. She can even acquire session keys of accepted entity instances. Our formal model of security for passwordauthenticated key exchange protocols is based on that of [2]. In the following, we review the operations of the adversary and formulate the definition of security. For details of the security model, we refer the readers to [2]. 
InitializATION. Let $I$ denote the identities of the protocol participants. Elements of $I$ will often be denoted $A$ and $B$ (Alice and Bob). We emphasis that $A$ and $B$ are variables ranging over $I$ and not fixed members of $I$. Each pair of entities, $A, B \in I$, are assigned a password $w$ which is randomly selected from the password space $\mathcal{D}$. The initialization process may also specify a set of cryptographic functions (e.g., hash functions) and establish a number of cryptographic parameters.

Running the Protocol. Mathematically, a protocol $\Pi$ is a probabilistic polynomial-time algorithm which determines how entities behave in response to received message. For each entity, there may be multiple instances running the protocol in parallel. We denote the $i$-th instance of entity $A$ as $\Pi_{A}^{i}$. The adversary $\mathcal{A}$ can make queries to any instance; she has an endless supply of $\Pi_{A}^{i}$ oracles $(A \in I$ and $i \in \mathbb{N})$. In response to each query, an instance updates its internal state and gives its output to the adversary. At any point in time, the instance may accept and possesses a session key $s k$, a session id sid, and a partner id pid. The query types, as defined in [2], include:

- Send $(A, i, M)$ : This sends message $M$ to instance $\Pi_{A}^{i}$. The instance executes as specified by the protocol and sends back its response to the adversary. Should the instance accept, this fact, as well as the session id and partner id will be made visible to the adversary.

- Execute $(A, i, B, j)$ : This call carries out an honest execution between two instances $\Pi_{A}^{i}$ and $\Pi_{B}^{j}$, where $A, B \in I, A \neq B$ and instances $\Pi_{A}^{i}$ and $\Pi_{B}^{j}$ were not used before. At the end of the execution, a transcript is given to the adversary, which logs everything an adversary could see during the execution (for details, see 2]).

- Reveal $(A, i)$ : The session key $s k_{A}^{i}$ of $\Pi_{A}^{i}$ is given to the adversary.

- Test $(A, i)$ : The instance $\Pi_{A}^{i}$ generates a random bit $b$ and outputs its session key $s k_{A}^{i}$ to the adversary if $b=1$, or else a random session key if $b=0$. This query is allowed only once, at any time during the adversary's execution.

- Oracle $(M)$ : This gives the adversary oracle access to a function $h$, which is selected at random from some probability space $\Omega$. The choice of $\Omega$ determines whether we are working in the standard model, or in the random-oracle model (see [2] for further explanations).

Note that the Execute query type can be implemented by using the Send query type. The Execute query type reflects the adversary's ability to passively eavesdrop on protocol execution. As well shall see, the adversary shall learn nothing about the password or the session key from such oracle calls. The Send query type allows the adversary to interact with entity instances and to carry out an active man-in-the-middle attack on the protocol execution.

Definition. Let $\Pi_{A}^{i}$ and $\Pi_{B}^{i}, A \neq B$, be a pair of instances. We say that $\Pi_{A}^{i}$ and $\Pi_{B}^{i}$ are partnered if both instances have accepted and hold the same session id sid and the same session key sk. Here, we define the sid of $\Pi_{A}^{i}$ ( or $\Pi_{B}^{i}$ ) as the concatenation of all the messages sent and received by $\Pi_{A}^{i}$ ( or $\Pi_{B}^{i}$ ). We say that $\Pi_{A}^{i}$ is fresh if: i) it has accepted; and ii) a Reveal query has not been called either 
on $\Pi_{A}^{i}$ or on its partner (if there is one). With these notions, we now define the advantage of the adversary $\mathcal{A}$ in attacking the protocol. Let Succ denote the event that $\mathcal{A}$ asks a single Test query on a fresh instance, outputs a bit $b^{\prime}$, and $b^{\prime}=b$, where $b$ is the bit selected during the Test query. The advantage of the adversary $\mathcal{A}$ is defined as $\operatorname{Adv}_{\mathcal{A}}^{a k e}=2 \operatorname{Pr}($ Succ $)-1$.

It is clear that a polynomial-time adversary $\mathcal{A}$ can always gain an advantage close to 1 if we do not limit her capability to perform on-line password-guessing attacks. In such an attack, the adversary picks a password $\pi$ as her guess and then impersonates as an instance $\Pi_{A}^{i}$ to start the protocol towards another instance $\Pi_{B}^{j}$. By observing the decision of $\Pi_{B}^{j}$ (i.e., accepts or rejects), the adversary can test the correctness of the guessed password $\pi$. Furthermore, by analyzing, offline, the transcript of the execution, the adversary may be able to test passwords other than $\pi$. For a secure protocol, we expect that the adversary can only test a single password in each on-line password-guessing attack. As suggested in [10, we use the Send query type to count the number of on-line guesses performed by the adversary. We only count one Send query for each entity instance, that is, if the adversary sends two Send queries to an entity instance, it should still count as a single password guess. Based on this idea, we have the following definition of secure password-authenticated key exchange protocol, which is the same as in 10 .

Definition 1. A protocol $\Pi$ is called a secure password-authenticated key exchange protocol if for every polynomial-time adversary $\mathcal{A}$ that makes at most $Q_{\text {send }}\left(Q_{\text {send }} \leq|\mathcal{D}|\right)$ queries of Send type to different instances, the following two conditions are satisfied:

(1) Except with negligible probability, each oracle call Execute $(A, i, B, j)$ produces a pair of partnered instances $\Pi_{A}^{i}$ and $\Pi_{B}^{j}$.

(2) $\operatorname{Adv}_{\mathcal{A}}^{\text {ake }} \leq Q_{\text {send }} /|\mathcal{D}|+\epsilon$, where $|\mathcal{D}|$ denotes the size of the password space and $\epsilon$ is a negligible function of security parameters.

The first condition basically says that when the adversary carries out an honest execution between two instances $\Pi_{A}^{i}$ and $\Pi_{B}^{j}(A \neq B)$, both instances accept and hold the same session key and the same session id. The second condition means that the advantage of the adversary is at most negligibly more than $Q_{\text {send }} /|\mathcal{D}|$ if she sends at most $Q_{\text {send }}$ queries of Send type to different entity instances, or equivalently, if she interacts on-line with at most $Q_{\text {send }}$ entity instances using the Send query type.

\section{Password Enabled Key Exchange Protocol}

In this section, we present a new protocol, called Password Enabled Key Exchange Protocol, or simply, PEKEP. In the protocol PEKEP, there are two entities, Alice and Bob, who share a password $w$ drawn at random from the password space $\mathcal{D}$ and Alice has also generated a pair of RSA keys $n, e$ and $d$, where $n$ is a large positive integer (e.g., $n>2^{1023}$ ) equal to the product of two primes of 
(roughly) the same size, $e$ is a positive integer relatively prime to $\phi(n)$, and $d$ is a positive integer such that $e d \equiv 1(\bmod \phi(n))$. The encryption function is define by $E(x)=x^{e} \bmod n, x \in \mathbb{Z}_{n}$. The decryption function is $D(x)=x^{d} \bmod n$. For a positive integer $m$, we define $E^{m}$ recursively as $E^{m}(x)=E\left(E^{m-1}(x)\right)=$ $x^{e^{m}} \bmod n$. Likewise, $D^{m}(x)=D\left(D^{m-1}(x)\right)=x^{d^{m}} \bmod n$. Before describing the protocol, let's review some of the facts of number theory.

Let $a$ be a positive integer relatively prime to $n$, we say that $a$ is an $e$-th power residue of $n$ if the congruence $x^{e} \equiv a(\bmod n)$ has a solution in $\mathbb{Z}_{n}^{*}$. Let $g$ be a positive integer relatively prime to $n$. The least positive integer $i$ such that $g^{i} \equiv 1(\bmod n)$ is called the order of $g$ modulo $n$. If the order of $g$ is equal to $\phi(n)$, then $g$ is called a primitive root of $n$. It is known (see [1,23) that a positive integer $n, n>1$, possesses a primitive root if and only if $n=2,4, p^{t}$ or $2 p^{t}$, where $p$ is an odd prime and $t$ is a positive integer. If $n$ has a primitive root $g$, then a positive integer $a$ relatively prime to $n$ can be represented as $a=g^{i}$

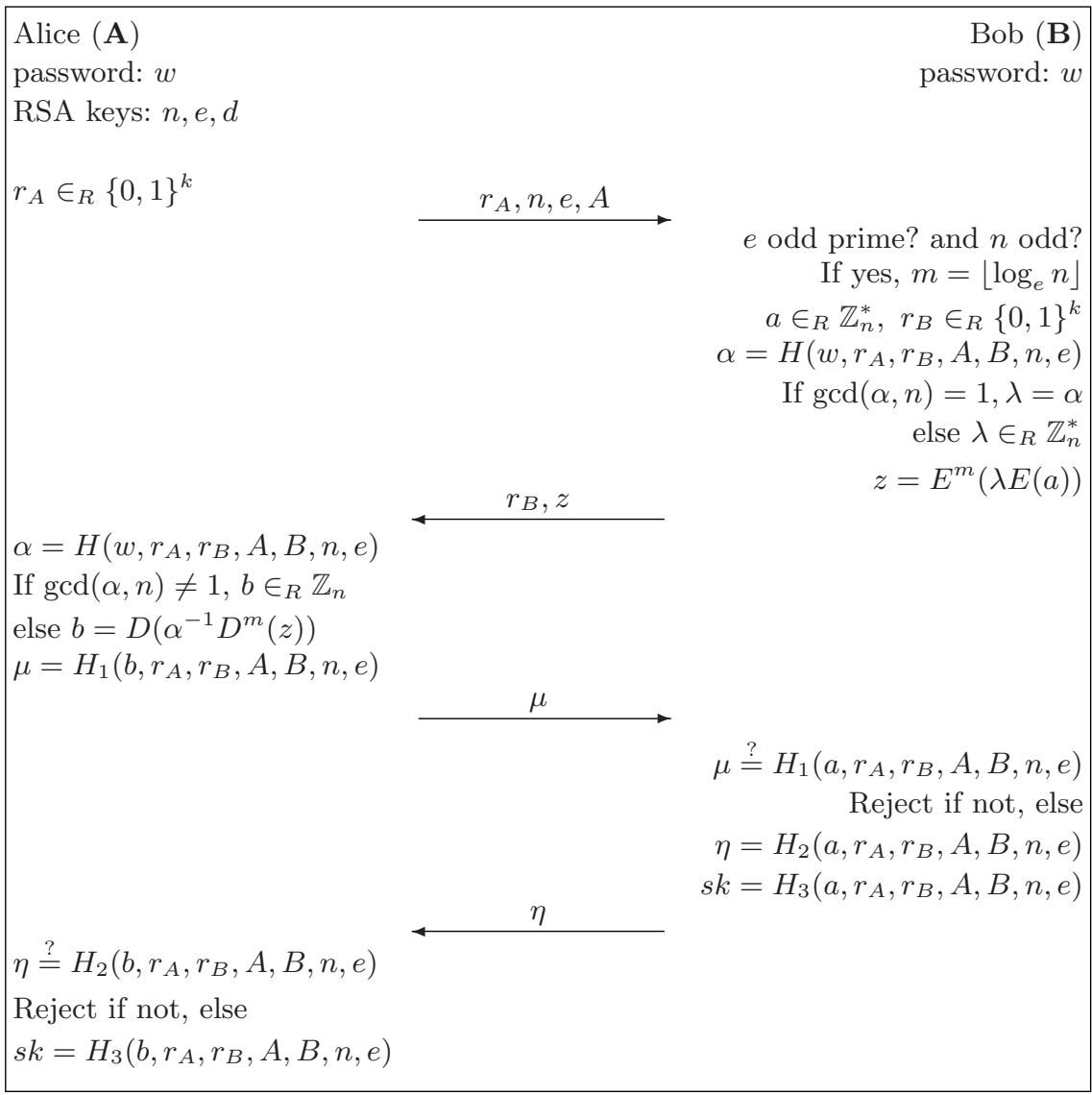

Fig. 1. Password Enabled Key Exchange Protocol (PEKEP) 
$\bmod n, 0 \leq i \leq \phi(n)-1$. The integer $i$ is called the index of a to the base $g$ modulo $n$, and is denoted by $\operatorname{ind}_{g} a$.

Define hash functions $H_{1}, H_{2}, H_{3}:\{0,1\}^{*} \rightarrow\{0,1\}^{k}$ and $H:\{0,1\}^{*} \rightarrow \mathbb{Z}_{n}$, where $k$ is a security parameter, e.g., $k=160$. Note that $H$ can be implemented using a standard hash function $h:\{0,1\}^{*} \rightarrow\{0,1\}^{\ell}$, where $\ell$ is the length of $n$, i.e., $\ell=\left\lceil\log _{2} n\right\rceil$. On input $x, H(x)=h(x)$, if $h(x)<n$, and $H(x)=h(x)-\lceil n / 2\rceil$ if else. The protocol PEKEP is described in Fig. 1. Alice starts the protocol by sending her public key $(n, e)$ and a random number $r_{A} \in_{R}\{0,1\}^{k}$ to Bob. Bob verifies if $e$ is an odd prime and $n$ is an odd integer. Bob may also verify that the integer $n$ is large enough, e.g., $n>2^{1023}$. If $e$ is not an odd prime or $n$ is not an odd integer, Bob rejects; otherwise, Bob computers an integer $m=\left\lfloor\log _{e} n\right\rfloor$ and selects two random numbers $a \in \in_{R} \mathbb{Z}_{n}^{*}$, and $r_{B} \in_{R}\{0,1\}^{k}$. Bob then computes $\alpha=H\left(w, r_{A}, r_{B}, A, B, n, e\right)$ and checks if $\operatorname{gcd}(\alpha, n)=1$. If $\operatorname{gcd}(\alpha, n) \neq 1$, Bob assigns a random number of $\mathbb{Z}_{n}^{*}$ to $\lambda$; otherwise, Bob assigns $\alpha$ to $\lambda$. Next, Bob computes $z=E^{m}(\lambda E(a))$ and sends $r_{B}$ and $z$ to Alice. Subsequently, Alice computes $\alpha$ using her password $w$ and checks if $\alpha$ and $n$ are relatively prime. If $\operatorname{gcd}(\alpha, n) \neq 1$, Alice assigns a random number of $\mathbb{Z}_{n}$ to the variable $b$. If $\operatorname{gcd}(\alpha, n)=1$ and $z$ is an $e^{m}$-th power residue of $n$, Alice sets $b=D\left(\alpha^{-1} D^{m}(z)\right)$. Next, Alice and Bob authenticate each other using $a$ and $b$ and generate a session key $s k$ upon successful authentication.

Note that, when $e$ is a prime number larger than $n$, Bob sets $m=0$. In this case, the run of PEKEP is nearly identical to that of SNAPI. In the protocol PEKEP, Bob only verifies if the public exponent $e$ is an odd prime and the RSA modulus $n$ is an odd integer; Bob does not verify if $e$ is relatively prime to $\phi(n)$. This may foster the so-called $e$-residue attack as described in [5, 22]. In the $e$-residue attack, an adversary, say, Eva, selects $\pi_{0} \in \mathcal{D}$ as her guess of Alice's password. She also selects an odd prime number $e$ and an odd integer $n$ such that $e \mid \phi(n)$, i.e., $(n, e)$ is not a valid RSA public key. Then Eva impersonates as Alice and starts the protocol PEKEP by sending $r_{E}, n, e, A$ to Bob, where $r_{E} \in\{0,1\}^{k}$ is a random number generated by Eva. After receiving $r_{B}$ and $z$ from Bob, Eva computes $\mu$ and sends it back to Bob. If Bob accepts, then Eva has a successful guess of Alice's password (i.e., $\pi_{0}$ ). If Bob rejects, on the other hand, Eva excludes her guess $\pi_{0}$ from the password space $\mathcal{D}$. Furthermore, Eva may exclude more passwords by repeating, off-line, the following three steps:

1) Eva selects a password $\pi$ from $\mathcal{D}$.

2) Eva computes $\alpha=H\left(\pi, r_{E}, r_{B}, A, B, n, e\right)$.

3) Eva tests if $\operatorname{gcd}(\alpha, n)=1$. If not, Eva returns to step 1; otherwise, Eva verifies if the congruence $\left(\alpha x^{e}\right)^{e^{m}} \equiv z(\bmod n)$ has a solution in $\mathbb{Z}_{n}^{*}$. If the congruence has a solution, Eva returns to step 1. If the congruence has no solution in $\mathbb{Z}_{n}^{*}$, then Eva knows that $\pi$ is not the password of Alice. Next, Eva excludes $\pi$ from $\mathcal{D}$ and returns to step 1 .

We say that Eva succeeds if she can exclude more than one password in the $e$-residue attack as described above. In the following, we show that the protocol PEKEP is secure against $e$-residue attack. 
Theorem 1. Let $n, n>1$, be an odd integer with prime-power factorization $n=p_{1}^{a_{1}} p_{2}^{a_{2}} \ldots p_{r}^{a_{r}}$. Let $m$ be a non-negative integer and e an odd prime such that for any prime-power $p_{i}^{a_{i}}$ of the factorization of $n, e^{m+1} \nmid \phi\left(p_{i}^{a_{i}}\right), 1 \leq i \leq r$. If $z$ is an $e^{m}$-th power residue of $n$, then for any $\lambda \in \mathbb{Z}_{n}^{*}$, the congruence $\left(\lambda x^{e}\right)^{e^{m}} \equiv$ $z(\bmod n)$ has a solution in $\mathbb{Z}_{n}^{*}$.

Proof. To prove that $\left(\lambda x^{e}\right)^{e^{m}} \equiv z(\bmod n)$ has a solution in $\mathbb{Z}_{n}^{*}$, we only need to prove that, for each prime power $p_{i}^{a_{i}}$ of the factorization of $n$, the following congruence

$$
\left(\lambda x^{e}\right)^{e^{m}} \equiv z\left(\bmod p_{i}^{a_{i}}\right)
$$

has a solution in $\mathbb{Z}_{p_{i}^{a_{i}}}^{*}$.

Let $n_{i}=p_{i}^{a_{i}}, 1 \leq i \leq r$. Then $\phi\left(n_{i}\right)=p_{i}^{a_{i}-1}\left(p_{i}-1\right)$. Since $n$ is odd, $p_{i}$ is an odd prime. Thus, the integer $n_{i}$ possesses a primitive root. Let $g$ be a primitive root of $n_{i}$, that is, $g^{\phi\left(n_{i}\right)}=1 \bmod n_{i}$, and for any $0 \leq i, j \leq \phi\left(n_{i}\right)-1, i \neq j$, $g^{i} \neq g^{j} \bmod n_{i}$. Let $\operatorname{gcd}\left(e^{m}, \phi\left(n_{i}\right)\right)=e^{c}, 0 \leq c \leq m$. We consider the following two cases:

(1) If $c=0$, then $e$ and $\phi\left(n_{i}\right)$ are relatively prime. In this case, it is clear that the congruence $\left(\lambda x^{e}\right)^{e^{m}} \equiv z\left(\bmod n_{i}\right)$ has a unique solution in $\mathbb{Z}_{n_{i}}^{*}$.

(2) Next, we consider the case that $1 \leq c \leq m$. Since $z$ is an $e^{m}$-th power residue of $n$, the congruence $y^{e^{m}} \equiv z(\bmod n)$ has solutions in $\mathbb{Z}_{n}^{*}$. By the Chinese Remainder Theorem, the following congruence

$$
y^{e^{m}} \equiv z\left(\bmod n_{i}\right)
$$

has solutions in $\mathbb{Z}_{n_{i}}^{*}$. Let ind $z$ denote the index of $z$ to the base $g$ modulo $n_{i}$ and let $y \in \mathbb{Z}_{n_{i}}^{*}$ be a solution of $(2)$, then $g^{e^{m} \operatorname{ind}_{g} y-\operatorname{ind}_{g} z} \equiv 1\left(\bmod n_{i}\right)$. Since the order of $g$ modulo $n_{i}$ is $\phi\left(n_{i}\right)$, we have

$$
e^{m} \operatorname{ind}_{g} y \equiv \operatorname{ind}_{g} z\left(\bmod \phi\left(n_{i}\right)\right)
$$

Also since $\operatorname{gcd}\left(e^{m}, \phi\left(n_{i}\right)\right)=e^{c}$, equation (3) has exactly $e^{c}$ incongruent solutions modulo $\phi\left(n_{i}\right)$ when taking $\operatorname{ind}_{g} y$ as variable. This indicates that equation (2) has $e^{c}$ solutions in $\mathbb{Z}_{n_{i}}^{*}$. Let $y_{0}$ denote one of the solutions of (2), then the $e^{c}$ incongruent solutions of $(3)$ are given by

$$
\operatorname{ind}_{g} y=\operatorname{ind}_{g} y_{0}+t \phi\left(n_{i}\right) / e^{c}\left(\bmod \phi\left(n_{i}\right)\right), \quad 0 \leq t \leq e^{c}-1 .
$$

For any $\lambda \in \mathbb{Z}_{n}^{*}$, we have

$$
\operatorname{ind}_{g} y-\operatorname{ind}_{g} \lambda \equiv \operatorname{ind}_{g} y_{0}-\operatorname{ind}_{g} \lambda+t \phi\left(n_{i}\right) / e^{c}\left(\bmod \phi\left(n_{i}\right)\right), \quad 0 \leq t \leq e^{c}-1 .
$$

Under the condition that $e^{m+1} \nmid \phi\left(n_{i}\right)$, it is clear that $e \nmid \phi\left(n_{i}\right) / e^{c}$. Hence, $\phi\left(n_{i}\right) / e^{c} \equiv 1(\bmod e)$. So, there exists an integer $t, 0 \leq t \leq e^{c}-1$, such that

$$
\operatorname{ind}_{g} y_{0}-\operatorname{ind}_{g} \lambda+t \phi\left(n_{i}\right) / e^{c} \equiv 0(\bmod e),
$$

which implies that there exists an integer $y \in \mathbb{Z}_{n_{i}}^{*}$, such that $y^{e^{m}} \equiv z\left(\bmod n_{i}\right)$ and $y \lambda^{-1}$ is an $e$-th power residue of $n_{i}$. Therefore, equation (1) has a solution in $\mathbb{Z}_{n_{i}}^{*}$, which proves the theorem. 
In PEKEP, Bob sets $m$ equal to $\left\lfloor\log _{e} n\right\rfloor$. Thus, for every prime-power $p_{i}^{a_{i}}$ of the factorization of $n$, we have $e^{m+1}>n \geq p_{i}^{a_{i}}$. By Theorem 1 , for any $\lambda \in \mathbb{Z}_{n}^{*}$, the congruence $\left(\lambda x^{e}\right)^{e^{m}} \equiv z(\bmod n)$ has a solution in $\mathbb{Z}_{n}^{*}$, where $z$ is the $e$-th power residue computed by Bob. Hence, by repeating (off-line) the three steps as described previously, Eva could not exclude any password from the space $\mathcal{D}$. So, the protocol PEKEP is secure against $e$-residue attacks. In Section 5, we will provide a formal analysis of PEKEP within the security model described in Section 2.

At the beginning of PEKEP, Bob needs to test the primality of the public exponent $e$ selected by Alice. When $e$ is small, e.g., $e=17$, the primality test only induces moderate overhead on Bob. When $e$ is large (e.g., $e>2^{512}$ ), however, the computational load for the primality test would be tremendous. In the following, we show that primality test of large public exponents by Bob could be avoided with slight modification of PEKEP. In the protocol PEKEP, Bob can actually select a small prime number $e^{\prime}\left(\right.$ e.g., $\left.e^{\prime}=3\right)$ and replaces Alice's public key $(n, e)$ by $\left(n, e^{\prime}\right)$, that is, Bob computes $m, \alpha, z, \eta, s k$ using $\left(n, e^{\prime}\right)$ instead of Alice's public key $(n, e)$. Theorem 1 demonstrates that the replacement does not lead to $e$-residue attacks, even if $e^{\prime}$ is not relatively prime to $\phi(n)$. So, when the public exponent $e$ received from Alice exceeds a threshold, Bob replaces $e$ by a smaller prime number $e^{\prime}\left(2<e^{\prime}<e\right)$ of his own choosing. Bob sends $r_{B}, z$, and $e^{\prime}$ to Alice in the second flow. After receiving $e^{\prime}$ from Bob, Alice tests if $e^{\prime}$ is relatively prime to $\phi(n)$. If $\operatorname{gcd}\left(e^{\prime}, \phi(n)\right) \neq 1$, Alice sends a random number $\mu \in\{0,1\}^{k}$ to Bob; Alice may select a smaller prime number for $e$ in the next communication session. If $\operatorname{gcd}\left(e^{\prime}, \phi(n)\right)=1$, Alice replaces her decryption key by $d^{\prime}$ and then proceeds as specified in Fig. 1, where $e^{\prime} d^{\prime} \equiv 1(\bmod \phi(n))$.

In each run of PEKEP, Bob computes $m+1$ encryptions using Alice's public key $(n, e)$, where $m=\left\lfloor\log _{e} n\right\rfloor$. The computation time for the $m+1$ encryptions is $\mathcal{O}\left(\left(\log _{2} n\right)^{3}\right)$, which means that the computational load on Bob is about the same as that in SNAPI. As discussed above, however, Bob does not have to perform primality test of large public exponents. Hence, the protocol PEKEP still improves on SNAPI by reducing the cost of primality test of RSA public exponent. Since Alice has knowledge of $\phi(n)$, she only needs to perform two decryptions in each run of PEKEP; one using the decryption key $d_{1}=d$ and another using the decryption key $d_{2}=d^{m} \bmod \phi(n)$. Note that the computational load on Bob is high even when $e$ is small. In Section 4 , we present a computationally-efficient key exchange protocol which greatly reduces the computational load on Bob.

\section{Computationally-Efficient Key Exchange Protocol}

In this section, we present a Computationally-Efficient Key Exchange Protocol (CEKEP), which is described in Fig. 2. The protocol CEKEP is based on PEKEP, but the number of encryptions performed by Bob is less than $\left\lfloor\log _{e} n\right\rfloor$, where $(n, e)$ is the public key of Alice. In the protocol CEKEP, Bob selects a small number $\varepsilon, 0<\varepsilon \leq 2^{-80}$, which determines the probability of a successful $e$-residue attack against the protocol CEKEP. Alice starts the protocol CEKEP 
by sending her public key $n, e$ and two random numbers $\rho, r_{A} \in_{R}\{0,1\}^{k}$ to Bob. Bob verifies if $e$ is an odd prime and $n$ is an odd integer. If not, Bob rejects. Else, Bob computers an integer $m$ based on $e$ and $\varepsilon$ as $m=\left\lceil\log _{e} \varepsilon^{-1}\right\rceil$. Then Bob selects a random number $\varrho \in_{R}\{0,1\}^{k}$ such that $\gamma=H(n, e, \rho, \varrho, A, B, m)$ is relatively prime to $n$. Bob sends $\varrho$ and $m$ to Alice. After receiving $\varrho$ and $m$, Alice computes $u=D^{m}(\gamma)$ and sends it back to Bob. Subsequently, Bob verifies if Alice has made the right decryption, i.e., $E^{m}(u)=\gamma$. If $\gamma \neq E^{m}(u)$, Bob rejects. Else, Alice and Bob executes the rest of the protocol as in PEKEP.

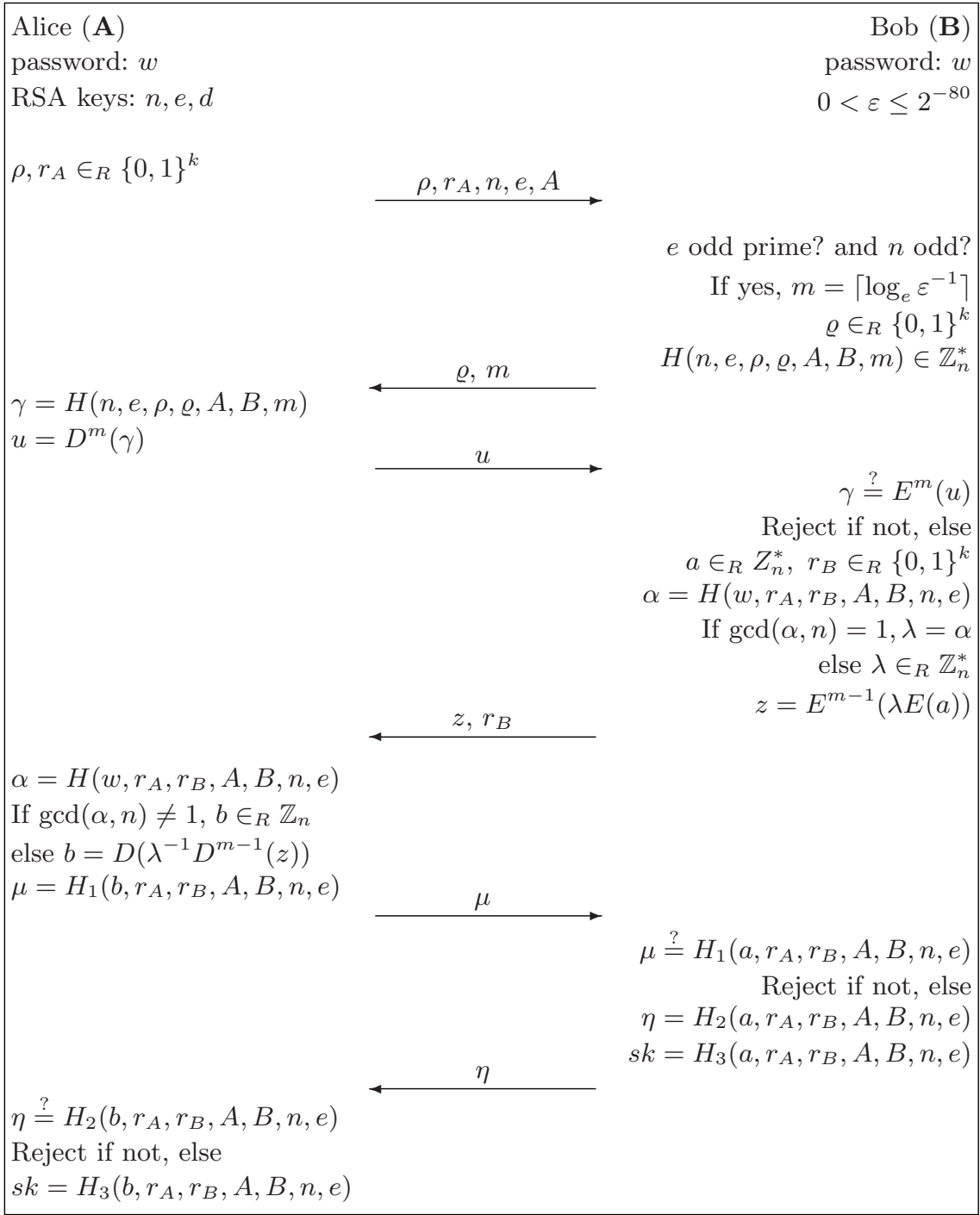

Fig. 2. Computationally-Efficient Key Exchange Protocol (CEKEP) 
A major difference between CEKEP and PEKEP is that the protocol CEKEP adds two additional flows between Alice and Bob. Through the two flows, Alice and Bob establish a random number $\gamma \in \mathbb{Z}_{n}^{*}$. Then Alice decrypts the random number $\gamma$ repeatedly $m$ times. If the $m$ repeated decryption is correct, i.e., $\gamma=E^{m}(u)$, then it can be concluded that, except with probability as small as $e^{-m}$, the integer $e^{m}$ does not divide $\phi\left(p_{i}^{a_{i}}\right)$ for every prime-power $p_{i}^{a_{i}}$ of the factorization of $n$.

Theorem 2. Let $n, n>1$, be an odd integer with prime-power factorization $n=p_{1}^{a_{1}} p_{2}^{a_{2}} \ldots p_{r}^{a_{r}}$. Let $m$ be a positive integer and $e$ an odd prime. If there exists a prime power, say $p_{i}^{a_{i}}$, of the factorization of $n$ such that $e^{m} \mid \phi\left(p_{i}^{a_{i}}\right)$, then for an integer $\gamma$ randomly selected from $\mathbb{Z}_{n}^{*}$, the probability that $\gamma$ is an $e^{m}$-th power residue of $n$ is less than or equal to $e^{-m}$.

Proof. Let $n_{i}=p_{i}^{a_{i}}$ be a prime power of the factorization of $n$ such that $e^{m}$ $\phi\left(n_{i}\right)$. Since $n$ is odd, $n_{i}$ possesses a primitive root. Let $g$ be a primitive root of $n_{i}$. For an integer $\gamma$ randomly selected from $\mathbb{Z}_{n}^{*}$, let ind $\operatorname{lof}_{g}$ denote the index of $\gamma$ to the base $g$ modulo $n_{i}$. Then $\gamma$ is an $e^{m}$-th power residue of $n_{i}$ if and only if the congruence $x^{e^{m}} \equiv \gamma\left(\bmod n_{i}\right)$ has a solution, or equivalently, if and only if

$$
g^{e^{m}} \operatorname{ind}_{g} x-\operatorname{ind}_{g} \gamma \equiv 1\left(\bmod n_{i}\right),
$$

which is equivalent to

$$
e^{m} \operatorname{ind}_{g} x \equiv \operatorname{ind}_{g} \gamma\left(\bmod \phi\left(n_{i}\right)\right) .
$$

Since $e^{m} \mid \phi\left(n_{i}\right), \gamma$ is an $e^{m}$-th power residue of $n_{i}$ if and only if $e^{m} \mid \operatorname{ind}_{g} \gamma$. Let $n_{i}^{\prime}=n / n_{i}$, then $n_{i}$ and $n_{i}^{\prime}$ are relatively prime. For any integer $\beta \in$ $\mathbb{Z}_{n}^{*}$, it is clear that $\beta \bmod n_{i}$ and $\beta \bmod n_{i}^{\prime}$ are integers of $\mathbb{Z}_{n_{i}}^{*}$ and $\mathbb{Z}_{n_{i}^{\prime}}^{*}$, respectively. On the other hand, for two integers $\alpha_{1} \in \mathbb{Z}_{n_{i}}^{*}$ and $\alpha_{2} \in \mathbb{Z}_{n_{i}^{\prime}}^{*}$, by the Chinese Remainder Theorem, there is an unique integer $\alpha \in \mathbb{Z}_{n}^{*}$, such that $\alpha \equiv \alpha_{1}\left(\bmod n_{i}\right)$, and $\alpha \equiv \alpha_{2}\left(\bmod n_{i}^{\prime}\right)$. So, the number of integers $\alpha \in \mathbb{Z}_{n}^{*}$ which satisfy the congruence $\alpha \equiv \alpha_{1}\left(\bmod n_{i}\right)$ is $\phi\left(n_{i}^{\prime}\right)$. If $\gamma$ is randomly selected from $\mathbb{Z}_{n}^{*}$, then for any integer $s, 0 \leq s \leq \phi\left(n_{i}\right)-1$, we have

$$
\operatorname{Pr}\left(g^{s}=\gamma \bmod n_{i}\right)=\phi\left(n_{i}^{\prime}\right) / \phi(n)=1 / \phi\left(n_{i}\right),
$$

which implies that $\operatorname{Pr}\left(\operatorname{ind}_{g} \gamma=s\right)=1 / \phi\left(n_{i}\right)$. Hence,

$$
\begin{aligned}
\operatorname{Pr}\left(e^{m} \mid \operatorname{ind}_{g} \gamma\right) & =\sum_{e^{m} \mid s, 0 \leq s<\phi\left(n_{i}\right)} \operatorname{Pr}\left(\operatorname{ind}_{g} \gamma=s\right) \\
& =\phi\left(n_{i}\right) e^{-m} / \phi\left(n_{i}\right) \\
& =e^{-m}
\end{aligned}
$$

which indicates that, for an integer $\gamma$ randomly selected from $\mathbb{Z}_{n}^{*}$, the probability that $\gamma$ is an $e^{m}$-th power residue of $n_{i}$ is equal to $e^{-m}$. So, the probability that $\gamma$ is an $e^{m}$-th power residue of $n$ does not exceed $e^{-m}$. 
Theorem 2 demonstrates that, if there exits a prime-power $p_{i}^{a_{i}}$ of the factorization of $n$ such that $e^{m} \mid \phi\left(p_{i}^{a_{i}}\right)$, then for a random number $\gamma \in \mathbb{Z}_{n}^{*}$, the probability that Alice can decrypt $\gamma$ repeatedly $m$ times is less than or equal to $e^{-m}$. If the number $u$ received from Alice satisfies the equation $E^{m}(u)=u^{e^{m}}=$ $\gamma \bmod n$, i.e., $\gamma$ is an $e^{m}$-power residue of $n$, then Bob is ensured with probability greater than or equal to $1-e^{-m}$ that, for every prime-power $p_{i}^{a_{i}}$ of the factorization of $n, e^{m} \nmid \phi\left(p_{i}^{a_{i}}\right)$. Since $m=\left\lceil\log _{e} \varepsilon^{-1}\right\rceil, e^{-m} \leq \varepsilon$. By Theorem 1 , it is clear that the probability for an adversary to launch a successful $e$-residue attack against CEKEP is upper-bounded by $\varepsilon$.

In the protocol CEKEP, Alice proves to Bob in an interactive manner (via flow 2 and flow 3) that for every prime-power $p_{i}^{a_{i}}$ of the factorization of $n$, $e^{m} \nmid \phi\left(p_{i}^{a_{i}}\right)$. In the interactive procedure, however, only one decrypted message is sent from Alice to Bob. The communication overhead on Alice and Bob is greatly reduced in comparison with that in 27, 24, 9. In CEKEP, the computational burden on Bob includes two modulo exponentiations, i.e., $u^{e^{m}} \bmod n$ and $\left(\lambda a^{e}\right)^{e^{m-1}} \bmod n$, where $m=\left\lceil\log _{e} \varepsilon^{-1}\right\rceil$. When $e<\varepsilon^{-1}$, each modulo exponentiation has an exponent consisting of $\mathcal{O}\left(\left\lceil\log _{2} \varepsilon^{-1}\right\rceil\right)$ bits. The computation time for the two modulo exponentiations is $\mathcal{O}\left(2\left(\log _{2} \varepsilon^{-1}\right)\left(\log _{2} n\right)^{2}\right)$. If $\varepsilon^{-1} \ll n$, then the computational load on Bob is greatly reduced in CEKEP in comparison with that in PEKEP (or in SNAPI). The parameter $\varepsilon$ determines the computational load on Bob. It also determines the level of security against $e$-residue attacks. In practice, Bob can make a trade-off between the computational load and the security level offered by the protocol. When $\varepsilon=2^{-80}$, for example, Bob needs to compute two modular exponentiation, each having an exponent of about 80 bits. In this case, the computational load on Bob is lighter than that in a Diffie-Hellman based password-authenticated key exchange protocol. In the Diffie-Hellman based EKE variant, for example, Bob needs to compute two modular exponentiation, each having an exponent of at least 160 bits.

\section{$5 \quad$ Formal Security Analysis}

In this section, we analyze the security of PEKEP and CEKEP within the formal model of security given in Section 2. Our analysis is based on the random-oracle model of Bellare and Rogaway 4. In this model, a hash function is modeled as an oracle which returns a random number for each new query. If the same query is asked twice, identical answers are returned by the oracle. In our analysis, we also assume the intractability of the RSA problem.

RSA Assumption: Let $\ell$ be the security parameter of RSA. Let key generator $G E$ define a family of RSA functions, i.e., $(e, d, n) \leftarrow G E\left(1^{\ell}\right)$, where $n$ is the product of two primes of the same size, $\operatorname{gcd}(e, \phi(n))=1$, and $e d \equiv 1(\bmod$ $\phi(n))$. For any probabilistic polynomial-time algorithm $\mathcal{C}$ of running time $t$, the following probability

$\operatorname{Adv}_{\mathcal{C}}^{r s a}(t)=\operatorname{Pr}\left(x^{e}=c \bmod n:(e, d, n) \leftarrow G E\left(1^{\ell}\right), c \in_{R}\{0,1\}^{\ell}, x \leftarrow \mathcal{C}\left(1^{\ell}, c, e, n\right)\right)$ 
is negligible. In the following, we use $\operatorname{Adv}^{r s a}(t)$ to denote $\max _{\mathcal{C}}\left\{\operatorname{Adv}_{\mathcal{C}}^{r s a}(t)\right\}$, where the maximum is taken over all polynomial-time algorithms of running time $t$.

Theorem 3. Let $\mathcal{A}$ be an adversary which runs in time $t$ and makes $Q_{\text {send }}$, $Q_{\text {send }} \leq|\mathcal{D}|$, queries of type Send to different instances. Then the adversary's advantage in attacking the protocol PEKEP is bounded by

$\operatorname{Adv}_{\mathcal{A}}^{\text {ake }} \leq \frac{Q_{\text {send }}}{|\mathcal{D}|}+\left(Q_{\text {execute }}+3 Q_{\text {send }}\right) \operatorname{Adv}^{r s a}(\mathcal{O}(t))+\mathcal{O}\left(\frac{\left(Q_{\text {execute }}+2 Q_{\text {send }}\right) Q_{\text {oh }}}{2^{k}}\right)$,

where $Q_{\text {execute }}$ denotes the number of queries of type Execute and $Q_{\text {oh }}$ denotes the number of random oracle calls.

We prove Theorem 3 through a series of hybrid experiments. In each experiment, we modify the way session keys are chosen for instances involved in protocol execution. We start by choosing random session keys (not output by random oracles) for instances for which the Execute oracle is called. We then proceed to choose random session keys for instances for which the Send oracle is called. These instances are gradually changed over five hybrid experiments and in the last hybrid experiment, all the session keys are chosen uniformly at random. Thus, the adversary $\mathcal{A}$ can not distinguish them from random. We denote these hybrid experiments by $P_{0}, P_{1}, \ldots, P_{4}$ and by $\operatorname{Adv}\left(\mathcal{A}, P_{i}\right)$ the advantage of $\mathcal{A}$ when participating in experiment $P_{i}$. The hybrid experiment $P_{0}$ describes the real adversary attack. For $0 \leq i \leq 3$, we show that the difference between $\operatorname{Adv}\left(\mathcal{A}, P_{i}\right)$ and $\operatorname{Adv}\left(\mathcal{A}, P_{i+1}\right)$ is negligible. We bound the advantage of $\mathcal{A}$ in $P_{4}$ by $Q_{\text {send }} /|D|+\epsilon$. Hence, the advantage of $\mathcal{A}$ in $P_{0}$ (i.e., in the real attack) is bounded by $Q_{\text {send }} /|D|+\epsilon$. Due to lack of space, the proof of Theorem 3 is omitted and can be found in the full version of this paper 28.

It is easy to check that the protocol PEKEP satisfies the first condition of Definition 1. Theorem 3 indicates that the protocol PEKEP also satisfies the second condition of Definition 1 and hence is a secure password-authenticated key exchange protocol. Similarly, we can also show that the protocol CEKEP satisfies the two conditions of Definition 1. In summary, we have the following theorem 4 .

Theorem 4. Both protocols, PEKEP and CEKEP, are secure password authenticated key exchange protocols under the RSA assumption and the random oracle model.

We notice that the random oracle model in Theorem 4 is less desirable than a standard cryptographic assumption. To avoid the random oracle model, we could use the proof technique of [12, which require a public-key encryption scheme secure against chosen-ciphertext attacks. Unfortunately, the most commonly used RSA schemes (e.g. 3, 7,) which are secure against chosen-ciphertext attacks are also based on the random oracle model. Nevertheless, it is encouraging to see that efficient password-authenticated key exchange protocols with security proof in the random oracle model can be constructed without severe restriction on the public key of RSA. 


\section{Conclusion}

In this paper, we investigate the design of RSA-based password-authenticated key exchange protocols that do not restrict the size of RSA public exponent. Based on number-theoretic techniques, we develop a Password Enabled Key Exchange Protocol (PEKEP) which can use both large and small primes as RSA public exponent. We show that the protocol PEKEP is secure against $e$ residue attacks. We also provide a formal security analysis of PEKEP under the RSA assumption and the random oracle model. Based on PEKEP, we develop a computationally-efficient key exchange protocol to mitigate the burden on communication entities. Both protocols, PEKEP and CEKEP, do not require public parameters; Alice and Bob only need to establish a shared password in advance and do not need to establish other common parameters such as a prime number $p$ and a generator $g$ of the cyclic group modulo $p$. This is appealing in environments where entities have insufficient resources to generate or validate public parameters with certain properties, e.g., primality.

\section{References}

1. E. Bach and J. Shallit, Algorithmic Number Theory, vol. 1: Efficient Algorithms, MIT Press, 1997.

2. M. Bellare, D. Pointcheval, and P. Rogaway, Authenticated key exchange secure against dictionary attack, Advances in Cryptology - EUROCRYPT 2000 Proceedings, Lecture Notes in Computer Science, vol. 1807, Springer-Verlag, 2000, pp. 139-155.

3. M. Bellare and P. Rogaway, Optimal asymmetric encryption, Advances in Cryptology - EUROCRYPT '94 proceedings, Lecture Notes in Computer Science, vol. 950, Springer-Verlag, 1995, pp. 92-111.

4. M. Bellare and P. Rogaway, Entity Authentication and key distribution, Advances in Cryptology - CRYPTO'93 Proceedings, Lecture Notes in Computer Science, vol. 773, Springer-Verlag, 1994, pp. 22-26.

5. S. M. Bellovin and M. Merritt, Encrypted key exchange: Password-based protocols secure against dictionary attacks, Proc. of the IEEE Symposium on Research in Security and Privacy, Oakland, May 1992, pp. 72-84.

6. S. M. Bellovin and M. Merritt, Augmented encrypted key exchange: A passwordbased protocol secure against dictionary attacks and password file compromise, Proc. of the 1st ACM Conference on Computer and Communications Security, ACM, November 1993, pp. 244-250.

7. D. Boneh, Simplified OAEP for the RSA and Rabin functions, Advances in Cryptology - CRYPTO 2001 Proceedings, Lecture Notes in Computer Science, vol. 2139, Springer-Verlag, 2001, pp. 275-291.

8. V. Boyko, P. MacKenzie, and S. Patel, Provably secure password authenticated key exchange using Diffie-Hellman, Advances in Cryptology - EUROCRYPT 2000 Proceedings, Lecture Notes in Computer Science, vol. 1807, Springer-Verlag, 2000, pp. 156-171.

9. D. Catalano, D. Pointcheval, and T. Pornin, IPAKE: Isomorphisms for Passwordbased Authenticated Key Exchange, to appear in CRYPTO 2004 Proceedings. 
10. R. Gennaro and Y. Lindell, A framework for password-based authenticated key exchange, Advances in Cryptology - EUROCRYPT 2003 Proceedings, Lecture Notes in Computer Science, vol. 2656, Springer-Verlag, 2003, pp.524-542.

11. O. Goldreich and Y. Lindell, Session-key generation using human passwords only, Advances in Cryptology - CRYPTO 2001 Proceedings, Lecture Notes in Computer Science, vol. 2139, Springer-Verlag, 2001, pp.408-432.

12. S. Halevi and H. Krawczyk, Public-key cryptography and password protocols, Proc. of the Fifth ACM Conference on Computer and Communications Security, 1998, pp. 122-131.

13. D. Jablon, Strong password-only authenticated key exchange, Computer Communication Review, ACM SIGCOMM, vol. 26, no. 5, 1996, pp. 5-26.

14. D. Jablon, http://www.integritysciences.com.

15. J. Katz, R. Ostrovsky, and M. Yung, Efficient password-authenticated key exchange using human-memorable passwords, Advances in Cryptology - EUROCRYPT 2001 Proceedings, Lecture Notes in Computer Science, vol. 2045, Springer-Verlag, 2001.

16. K. Kobara and H. Imai, Pretty-simple password-authenticated key-exchange under standard assumptions, IEICE Trans., vol. E85-A, no. 10, 2002, pp. 2229-2237.

17. T. Kwon, Authentication and key agreement via memorable passwords, Proc. Network and Distributed System Security Symposium, February 7-9, 2001.

18. S. Lucks, Open key exchange: How to defeat dictionary attacks without encrypting public keys, Proc. Security Protocol Workshop, Lecture Notes in Computer Science, vol. 1361, Springer-Verlag, 1997, pp. 79-90.

19. P. MacKenzie, S. Patel, and R. Swaminathan, Password-authenticated key exchange based on RSA, Advances in Cryptology-ASIACRYPT 2000 Proceedings, Lecture Notes in Computer Science, vol. 1976, Springer-Verlag, 2000, pp. 599-613.

20. A. Menezes, P. C. van Oorschot, S. A. Vanstone, Handbook of Applied Cryptography, CRC Press, 1997.

21. M. Nguyen and S. P. Vadhan, Simpler session-key generation from short random passwords, Proc. TCC 2004, Lecture Notes in Computer Science, vol. 2951, Springer-Verlag, 2004, pp. 428-445.

22. S. Patel, Number theoretic attacks on secure password schemes, Proc. IEEE Symposium on Security and Privacy, Oakland, California, May 5-7, 1997.

23. K. H. Rosen, Elementary Number Theory and Its Applications, 4th ed., Addison Wesley Longman, 2000.

24. D. Wong, A. Chan, and F. Zhu, More efficient password authenticated key exchange based on RSA, INDOCRYPT 2003 Proceedings, Lecture Notes in Computer Science, vol. 2904, Springer-Verlag, 2003, pp. 375-387.

25. T. Wu, The secure remote password protocol , Proc. Network and Distributed System Security Symposium, San Diego, March 1998, pp. 97-111.

26. T. Wu, A real-world analysis of Kerberos password security, Proc. Network and Distributed System Security Symposium, February 3-5, 1999.

27. F. Zhu, D. Wong, A. Chan, and R. Ye, RSA-based password authenticated key exchange for imbalanced wireless networks, Proc. Information Security Conference 2003 (ISC'02), Lecture Notes in Computer Science, vol. 2433, Springer-Verlag, 2002, pp.150-161.

28. M. Zhang, New approaches to password authenticated key exchange based on RSA, Cryptology ePrint Archive, Report 2004/033. 